\title{
Early Detection and Prediction of Cardiotoxicity in Chemotherapy-Treated Patients
}

\section{Citation}

Sawaya, Heloisa, Igal A. Sebag, Juan Carlos Plana, James L. Januzzi, Bonnie Ky, Victor Cohen, Sucheta Gosavi, et al. 2011. "Early Detection and Prediction of Cardiotoxicity in ChemotherapyTreated Patients." The American Journal of Cardiology 107 (9) (May): 1375-1380. doi:10.1016/ j.amjcard.2011.01.006. http://dx.doi.org/10.1016/j.amjcard.2011.01.006.

\section{Published Version}

doi:10.1016/j.amjcard.2011.01.006

\section{Permanent link}

http://nrs.harvard.edu/urn-3:HUL.InstRepos:29048910

\section{Terms of Use}

This article was downloaded from Harvard University's DASH repository, and is made available under the terms and conditions applicable to Other Posted Material, as set forth at http:// nrs.harvard.edu/urn-3:HUL.InstRepos:dash.current.terms-of-use\#LAA

\section{Share Your Story}

The Harvard community has made this article openly available.

Please share how this access benefits you. Submit a story.

\section{Accessibility}




\section{Early Detection and Prediction of Cardiotoxicity in Chemotherapy-Treated Patients}

Heloisa Sawaya, MD, PhDa, Igal A. Sebag, MD ${ }^{d}$, Juan Carlos Plana, MD ${ }^{f}$, James L. Januzzi, MD $^{\mathrm{a}}$, Bonnie Ky, MDg, Victor Cohen, MD $^{\mathrm{e}}$, Sucheta Gosavi, MD ${ }^{\mathrm{a}}$, Joseph R. Carver, MD ${ }^{\mathrm{g}}$, Susan E. Wiegers, MD ${ }^{g}$, Randolph P. Martin, MD ${ }^{h}$, Michael H. Picard, MDa ${ }^{a}$, Robert E. Gerszten, $\mathbf{M D}^{\mathrm{a}}$, Elkan F. Halpern, $\mathbf{P h D}^{\mathrm{c}}$, Jonathan Passeri, $\mathbf{M D}^{\mathrm{a}}$, Irene Kuter, $\mathbf{M D}^{\mathrm{b}}$, and Marielle Scherrer-Crosbie, MD, PhD ${ }^{\mathrm{a},{ }^{*}}$

${ }^{a}$ Cardiac Ultrasound Laboratory and Division of Cardiology, Massachusetts General Hospital and Harvard Medical School, Boston, Massachusetts

${ }^{b}$ Gillette Center for Breast Cancer, Massachusetts General Hospital and Harvard Medical School, Boston, Massachusetts

'Institute for Technology Assessment, Massachusetts General Hospital and Harvard Medical School, Boston, Massachusetts

dEchocardiography Laboratory and Cardiology Division, Sir Mortimer B. Davis-Jewish General Hospital and McGill University, Montreal, Quebec, Canada

eAbramson Cancer Center and Cardiology Division, Department of Medicine, Sir Mortimer B. Davis-Jewish General Hospital and McGill University, Montreal, Quebec, Canada

fDivision of Cardiology, University of Texas M.D. Anderson Cancer Center, Houston, Texas

gDivision of Cardiology, Hospital of the University of Pennsylvania, Philadelphia, Pennsylvania

hPiedmont Heart Institute, Atlanta, Georgia

\section{Abstract}

As breast cancer survival increases, cardiotoxicity associated with chemotherapeutic regimens such as anthracyclines and trastuzumab becomes a more significant issue. Assessment of the left ventricular (LV) ejection fraction fails to detect subtle alterations in LV function. The objective of this study was to evaluate whether more sensitive echocardiographic measurements and biomarkers could predict future cardiac dysfunction in chemotherapy-treated patients. Forty-three patients diagnosed with breast cancer who received anthracyclines and trastuzumab therapy underwent echocardiography and blood sampling at 3 time points (baseline and 3 and 6 months during the course of chemotherapy). The LV ejection fraction; peak systolic myocardial longitudinal, radial, and circumferential strain; echocardiographic markers of diastolic function; $\mathrm{N}$-terminal pro-B-type natriuretic peptide; and high-sensitivity cardiac troponin I were measured. Nine patients (21\%) developed cardiotoxicity ( 1 at 3 months and 8 at 6 months) as defined by the Cardiac Review and Evaluation Committee reviewing trastuzumab. A decrease in longitudinal strain from baseline to 3 months and detectable high-sensitivity cardiac troponin I at 3 months were independent predictors of the development of cardiotoxicity at 6 months. The LV ejection fraction, parameters of diastolic function, and $\mathrm{N}$-terminal pro-B-type natriuretic peptide did not predict cardiotoxicity. In conclusion, cardiac troponin plasma concentrations and longitudinal strain predict the development of cardiotoxicity in patients treated with anthracyclines and

(C) 2011 Elsevier Inc. All rights reserved.

*Corresponding author: Tel: 617-726-7686; fax: 617-726-8383. marielle@ crosbie.com (M. Scherrer-Crosbie). 
trastuzumab. The 2 parameters may be useful to detect chemotherapy-treated patients who may benefit from alternative therapies, potentially decreasing the incidence of cardiotoxicity and its associated morbidity and mortality.

The overall objective of this study was to assess whether early echocardiographic measurements of myocardial deformation and biomarkers (high-sensitivity troponin I [hsTnI] and N-terminal pro-B-type natriuretic peptide [NT-proBNP]) could predict the development of chemotherapy-induced cardiotoxicity in patients treated with anthracyclines and trastuzumab. Cardiotoxicity was defined according to recent guidelines (Cardiac Review and Evaluation Committee of trastuzumab-associated cardiotoxicity) as a reduction of the left ventricular ejection fraction (LVEF) of $\geq 5 \%$ to $<55 \%$ with symptoms of heart failure or an asymptomatic reduction of the LVEF of $\geq 10 \%$ to $<55 \%$. $^{1}$

\section{Methods}

Patients >18 years of age diagnosed with HER-2-overexpressing breast cancer and either scheduled to receive treatment including anthracyclines and trastuzumab or scheduled to receive trastuzumab after previous anthracycline treatment were eligible. Patients with LVEFs $<50 \%$ were excluded.

Patients were enrolled at 4 institutions. All patients signed informed consent forms, which were approved by the institutional review board of the participating institutions.

Patients were studied before chemotherapy (except 10 patients who had previously been treated with anthracyclines) and at 3 and 6 months of treatment, using questionnaires, echocardiography, and blood samples.

Transthoracic echocardiography was performed using the Vivid 7 or E9 (GE Healthcare, Milwaukee, Wisconsin). The same ultrasound machine was used to acquire all echocardiograms in each patient. All echocardiograms were analyzed in a core laboratory. The LVEF was calculated from the apical 4- and 2-chamber views using a modified Simpson's biplane method ${ }^{2}$ by a single observer (M.S.-C.). All other measurements were acquired by a single observer (H.S.) blinded to the results of the LVEF measurements. The 2 readers were blinded to the treatment that patients were receiving and to the time points during the study. Mitral E and A waves and annular velocities were measured. Left atrial volume was calculated from the apical 4- and 2-chamber views using the area-length method. To measure myocardial strain, 2-dimensional grayscale images were obtained in the parasternal short-axis view at the midpapillary level and the apical 4- and 2-chamber views. The timing of aortic valve closure was obtained using pulsed-wave Doppler traces. Peak systolic radial and circumferential strain were measured by averaging the peak systolic strain values in all 6 segments of the parasternal short-axis view (EchoPAC; GE Healthcare). In 5 patients for radial strain and 11 patients for circumferential strain, $>2$ of 6 segments could not be reproducibly analyzed at 1 time point. This was especially the case when the sonographer was unable to obtain a perfectly rounded parasternal short-axis view. The values of radial and circumferential strain were excluded for these patients. Furthermore, particularly in patients with left-sided breast cancer, the apex was difficult to visualize (inability to obtain standard apical views, chest pain, scar tissue, expanders). We estimate that in $60 \%$ to $70 \%$ of the patients, the apex was not analyzable at all time points. Hence, global peak systolic longitudinal strain was calculated by averaging the values of peak systolic strain in the basal and midwall segments of the 4- and 2-chamber views. Longitudinal, radial, and circumferential strain values were averaged over 3 cardiac cycles. The intraobserver variability for longitudinal strain reported as the mean error \pm SD of 10 measurements was $-0.14 \pm 1.1 \%$ in absolute values $(-0.58 \pm 6.5 \%$ in percentages $)$ and the 
interobserver variability as $0.5 \pm 1.5 \%$ in absolute values ( $2.7 \pm 7.9 \%$ in percentages). The variabilities for radial strain were $2 \pm 5 \%(2.5 \pm 9.6 \%)$ and $2.2 \pm 7.5 \%(4.4 \pm 14.4 \%)$, respectively. The intraobserver variability for circumferential strain was $0.53 \pm 2.82 \%$ in absolute values ( $2 \pm 13 \%$ in percentages), and the interobserver variability was $3.3 \pm 3.5 \%$ in absolute values $(19 \pm 21 \%$ in percentages $)$.

NT-proBNP and troponin I were measured using the Dimension Vista 500 Intelligent Laboratory System (Siemens Healthcare Diagnostics, Deerfield, Illinois), using a 1-step, homogenous, sandwich chemiluminescent immunoassay based on LOCI technology (Siemens Healthcare Diagnostics, Deerfield, Illinois). The limit of detection of NT-proBNP assay is $0.8 \mathrm{pg} / \mathrm{ml}$. Typical within-laboratory imprecision for the assay is $<3 \%$ coefficient of variation at NT-proBNP concentration from 120 to $5,000 \mathrm{pg} / \mathrm{ml}$. The highest value of NTproBNP reported in healthy subjects aged $<75$ years is $125 \mathrm{pg} / \mathrm{ml}$. The lower limit of detection of the troponin assay is $0.015 \mu \mathrm{g} / \mathrm{L}$, and the $10 \%$ coefficient of variation is $<0.04$ $\mu \mathrm{g} / \mathrm{L}$. All values of NT-proBNP $>125 \mathrm{pg} / \mathrm{ml}$ and of troponin $>0.015 \mu \mathrm{g} / \mathrm{L}$ were considered elevated.

Data are expressed as mean $\pm \mathrm{SD}$. All statistical analyses were performed using the JMP statistical package (SAS Institute Inc., Cary, North Carolina). Assuming a baseline peak systolic longitudinal strain in healthy patients of $19 \pm 5 \%^{3}$ and a rate of left ventricular dysfunction of $20 \%,{ }^{4}$ the analysis of 45 patients was expected to allow us to detect a decrease of $14 \%$ in longitudinal strain in the left ventricular dysfunction group with power of $80 \%$ and a $5 \%$ level of significance. Parameters of interest were compared at baseline and at 3 and 6 months in the population using a 1-way analysis of variance for repeated measures. Possible predictors of cardiotoxicity were tested using univariate nominal logistic regression. The primary hypothesis was to test the echocardiographic markers of systolic function and the biomarkers. Echocardiographic markers of diastolic function were also tested because they have been reported to decrease in patients treated with anthracyclines. A multiple nominal logistic regression model including longitudinal strain and troponin levels at 3 months was then applied to the univariate predictors. Because radial strain could not be reliably obtained in all patients, it was excluded from the model. A p value $<0.05$ was defined as significant.

\section{Results}

Forty-five eligible consecutive women were prospectively enrolled in the study. One woman withdrew from the study and 1 was withdrawn because of negative HER-2 status on a second analysis. Therefore, a total of 43 women participated in the study. Ten women received anthracyclines before entering the study. The median delay time between the anthracycline treatment and the initial study time point was 3.5 months (range 2 to 144). There was no difference in the baseline or changes in the clinical parameters, echocardiographic measurements, and biomarker levels between naive and previously treated women. All women were therefore analyzed together. Baseline characteristics of the patients are listed in Table 1.

At baseline, the LVEF and peak systolic longitudinal and radial strain were within the normal reported range of values ${ }^{3}$ (Table 2). When the group was analyzed as a whole, the LVEF decreased by an average of $8 \%$. This decrease was significant at 6 but not at 3 months. Longitudinal strain decreased by $11 \%$; this decrease was significant 3 months after the start of the study period. The decrease in longitudinal strain observed at 3 months was detected in the midwall $(p=0.008)$ and the lateral $(p=0.01)$ and anterior $(p=0.01)$ segments. Circumferential strain decreased by a mean of $15 \%$ at 6 months and was already decreased at 3 months. In contrast, radial strain did not decrease at 3 months when all 
women were studied. No change in hsTnI or NT-proBNP was noted in the course of the study.

Nine patients (21\%) met the criteria for cardiotoxicity. Cardiotoxicity was detected at the 3month time point in 1 patient (immediately after anthracyclines), whereas 8 other patients developed cardiotoxicity at the 6-month time point. There were no differences in any of the echocardiographic or blood markers at baseline between patients who did or did not develop cardiotoxicity. Importantly, the change of the LVEF from baseline to 3 months was not predictive of later cardiotoxicity ( $\mathrm{p}=0.19$; Table 3 ). This was also true when change in the LVEF was considered a discrete change, and patients were separated by those who did or did not have decreases in the LVEF of $>5 \%$. The individual changes in the LVEF and longitudinal strain are shown in Figure 1 in patients who did or did not develop cardiotoxicity.

In contrast, decreases in longitudinal strain and radial strain and elevated hsTnI at 3 months were predictive of patients who developed cardiotoxicity at 6 months (Table 3 ).

Furthermore, the number of abnormal segments at 3 months (defined either as segments in which longitudinal strain decreased by $\geq 10 \%$ between baseline and 3 months of treatment or by absolute segmental values of strain less than the mean -2 SD value of the same segment at baseline in the entire cohort) was lower in patients who did not develop cardiotoxicity than in patients who did. The number of abnormal segments at 3 months was predictive of cardiotoxicity ( $p<0.01$ for both definitions). The decrease in circumferential strain was not predictive of later cardiotoxicity. Neither the change in NT-proBNP between baseline and 3 months nor an NT-proBNP level higher than normal limits at 3 months predicted cardiotoxicity. None of the echocardiographic indexes of diastolic function at 3 months or their change between 0 and 3 months was predictive of cardiotoxicity at 6 months.

By multiple logistic regression analysis, elevated hsTnI at 3 months ( $\mathrm{p}<0.02)$ and a decrease in longitudinal strain between baseline and 3 months $(\mathrm{p}<0.02)$ remained independent predictors of later cardiotoxicity.

Longitudinal strain decreased by $>10 \%$ between 0 and 3 months in 14 patients (32\%). HsTnI was elevated at 3 months in 12 patients (28\%). The sensitivity, specificity, and positive and negative predictive values of hsTnI elevation and/or of a decrease of $>10 \%$ in longitudinal strain are reported in Table 4.

\section{Discussion}

In the present study, we report that in patients treated with a combination of anthracyclines and trastuzumab, an early decrease in myocardial strain or elevation in plasma troponin as detected with a high-sensitivity assay predicts the later occurrence of cardiotoxicity.

Subsequent cardiotoxicity is not clearly predicted by early changes in the LVEF or NTproBNP level.

Patients enrolled in our study experienced decreases in the LVEF of similar magnitude as those reported previously in patients treated with anthracyclines and trastuzumab. ${ }^{4-6}$ Peak systolic radial, longitudinal, and circumferential strain decreased before any LVEF decrease. Similarly, in 13 children and in 16 women treated with anthracyclines, investigators reported decreases in peak systolic longitudinal and radial strain during the treatment. ${ }^{7,8}$ In contrast, echocardiograms obtained before and after trastuzumab therapy did not reveal a decrease in myocardial strain. ${ }^{9}$ Although we reported that in a mouse model of anthracycline-induced cardiotoxicity, myocardial strain rate predicted later decrease in the LVEF and subsequent mortality, ${ }^{10}$ the value of an early measurement of myocardial strain in humans for the prediction of later cardiotoxicity has not been reported. 
Several factors may explain the increased sensitivity of strain compared to the LVEF in the detection of early cardiotoxicity found in our patients. As suggested by our findings, it is conceivable that the chemotherapy-induced cardiotoxicity has a regional pattern; the function of some myocardial segments may compensate for others, leading to a preserved LVEF, at least in the early stages. Another possible contributing factor to the higher sensitivity of strain compared to the LVEF may be variability. Strain measurement involves the averaging of the automated measurement of multiple segments, whereas LVEF assessment involves a tracing leading to 1 measurement. The variability (especially in the longitudinal dimension) may therefore be lower for strain than for the LVEF, particularly in patients with suboptimal images.

Using a high-sensitivity assay, we found that troponin I was elevated at 3 months of treatment in $28 \%$ of patients, suggesting that myocardial damage is frequent in chemotherapy-treated patients. Whereas Cardinale et a ${ }^{11}$ detected elevated troponin in $33 \%$ of patients after high doses of anthracyclines, more recent studies report less frequent troponin elevation and no value in predicting the decrease in LVEF after anthracyclines. ${ }^{12,13}$ In the present study, 1 sample obtained at 3 months of treatment was able to detect later LVEF decrease in 6 of 9 patients. Reconciling the results of previous studies, it is necessary to point out that the assay used in this study possesses an analytic precision that allows resolution of very small elevations in troponin I.

Neither the baseline value nor the change in NT-proBNP between 0 and 3 months was predictive of later decrease in the LVEF. Patients decreased their mitral annular velocities (E ') throughout the treatment $(p=0.004)$. This decrease was not accompanied by a decrease in the ratio of transmitral early peak velocity to early mitral annular velocity $\left(\mathrm{E} / \mathrm{E}^{\prime}\right)$ and may reflect subtle diastolic abnormalities rather than changes in filling pressures. This decrease was significant at the earliest time point ( 3 months) and is consistent with the early diastolic abnormalities reported in earlier studies. ${ }^{14-16}$

The follow-up period of the patients was 6 months; longer periods of follow-up will be necessary to determine whether the decrease of longitudinal strain or the increase in troponin is predictive of later cardiac events. The estimated sensitivity and specificity should also only be considered preliminary estimates considering the small number of patients.

The clinical implications of the present study are important. The reported findings demonstrate that myocardial strain and troponin, 2 noninvasive, easily performed measurements, recognize subtle myocardial alterations and predict later decrease in the LVEF in patients receiving anthracyclines and trastuzumab. The negative predictive value of the 2 tests allows the confident exclusion of cardiotoxicity detected by LVEF assessment 3 months later. Patients who do not have decreases of $>10 \%$ in their peak systolic longitudinal strain or elevated hsTnI levels have a 3\% probability of decreases in their LVEFs 3 months after the evaluation. In contrast, patients who either demonstrate decreases in longitudinal strain or elevations in hsTnI have a ninefold increase in risk for cardiotoxicity at 6 months compared to those with no changes in either of these markers. The presence of these predictive markers does not mandate cessation of a potentially lifesaving anticancer therapy. Rather, myocardial strain and hsTnI measurements may help target patients who could benefit from closer cardiac monitoring, earlier initiation of cardioprotective medical therapy, or less cardiotoxic novel tyrosine kinase inhibitors and anticancer drugs.

\section{Acknowledgments}

We would like to thank Laurie Farrell, RN, from the Cardiology Division for her help in the blood sampling and processing; Alisha Polewarczyk from the Gillette Center for Breast Cancer of Massachusetts General Hospital for her contribution in recruiting patients; Stephanie Fuoco, RN, from the Sir Mortimer B. Davis-Jewish General 
Hospital and McGill University and Jose Banchs, MD, Liza Sanchez, BS, and Mona Massey, RN, from the M.D. Anderson Cancer Center for coordinating patient enrollment; and Mary Lou Gantzer, PhD, and Joyce Melchior, BS (MTASCP), from Siemens Healthcare Diagnostics for doing the measurements of hsTnI and NT-proBNP.

Dr. Scherrer-Crosbie was supported by an investigator-initiated grant from the Susan G. Komen for the Cure Foundation, Dallas, Texas, a Claflin Distinguished Scholar Award, and a Clinical Innovation Award, Boston, Massachusetts. Dr. Ky was supported by the Kynett Focus Junior Faculty Investigator Award, Philadelphia, Pennsylvania.

Dr. Januzzi has received grant support from Roche Diagnostics GmbH, Mannheim, Germany, Siemens Medical Systems, Erlangen, Germany, and Critical Diagnostics, San Diego, California. Dr. Plana is on the speaker's bureau of GE Healthcare, Milwaukee, Wisconsin.

\section{References}

1. Martin M, Esteva FJ, Alba E, Khandheria B, Perez-Isla L, Garcia-Saenz JA, Marquez A, Sengupta P, Zamorano J. Minimizing cardiotoxicity while optimizing treatment efficacy with trastuzumab: review and expert recommendations. Oncologist. 2009; 14:1-11. [PubMed: 19147689]

2. Vuille, C.; Weyman, AE. Principles and Practice of Echocardiography. Philadelphia: Lea \& Febiger; 1994. Left ventricle I: general considerations, assessment of chamber size and function; $p$. 575-624.

3. Marwick TH, Leano RL, Brown J, Sun JP, Hoffmann R, Lysyansky P, Becker M, Thomas JD. Myocardial strain measurement with 2-dimensional speckle-tracking echocardiography: definition of normal range. J Am Coll Cardiol Cardiovasc Imaging. 2009; 2:80-84.

4. Tan-Chiu E, Yothers G, Romond E, Geyer CE Jr, Ewer M, Keefe D, Shannon RP, Swain SM, Brown A, Fehrenbacher L, Vogel VG, Seay TE, Rastogi P, Mamounas EP, Wolmark N, Bryant J. Assessment of cardiac dysfunction in a randomized trial comparing doxorubicin and cyclophosphamide followed by paclitaxel, with or without trastuzumab as adjuvant therapy in nodepositive, human epidermal growth factor receptor 2-overexpressing breast cancer: NSABP B-31. J Clin Oncol. 2005; 23:7811-7819. [PubMed: 16258083]

5. Bengala C, Zamagni C, Pedrazzoli P, Matteucci P, Ballestrero A, Da Prada G, Martino M, Rosti G, Danova M, Bregni M, Jovic G, Guarneri V, Maur M, Conte PF. Cardiac toxicity of trastuzumab in metastatic breast cancer patients previously treated with high-dose chemotherapy: a retrospective study. Br J Cancer. 2006; 94:1016-1020. [PubMed: 16570045]

6. Kelly H, Kimmick G, Dees EC, Collichio F, Gatti L, Sawyer L, Ivanova A, Dressler L, Graham ML, Carey LA. Response and cardiac toxicity of trastuzumab given in conjunction with weekly paclitaxel after doxorubicin/cyclophosphamide. Clin Breast Cancer. 2006; 7:237-243. [PubMed: 16942640]

7. Ganame J, Claus P, Eyskens B, Uyttebroeck A, Renard M, D’Hooge J, Gewillig M, Bijnens B, Sutherland GR, Mertens L. Acute cardiac functional and morphological changes after Anthracycline infusions in children. Am J Cardiol. 2007; 99:974-977. [PubMed: 17398195]

8. Jurcut R, Wildiers H, Ganame J, D’Hooge J, De Backer J, Denys H, Paridaens R, Rademakers F, Voigt JU. Strain rate imaging detects early cardiac effects of pegylated liposomal Doxorubicin as adjuvant therapy in elderly patients with breast cancer. J Am Soc Echocardiogr. 2008; 21:12831289. [PubMed: 19041569]

9. Hare JL, Brown JK, Leano R, Jenkins C, Woodward N, Marwick TH. Use of myocardial deformation imaging to detect preclinical myocardial dysfunction before conventional measures in patients undergoing breast cancer treatment with trastuzumab. Am Heart J. 2009; 158:294-301. [PubMed: 19619708]

10. Neilan TG, Jassal DS, Perez-Sanz TM, Raher MJ, Pradhan AD, Buys ES, Ichinose F, Bayne DB, Halpern EF, Weyman AE, Derumeaux G, Bloch KD, Picard MH, Scherrer-Crosbie M. Tissue Doppler imaging predicts left ventricular dysfunction and mortality in a murine model of cardiac injury. Eur Heart J. 2006; 27:1868-1875. [PubMed: 16717080]

11. Cardinale D, Sandri MT, Colombo A, Colombo N, Boeri M, Lamantia G, Civelli M, Peccatori F, Martinelli G, Fiorentini C, Cipolla CM. Prognostic value of troponin I in cardiac risk stratification of cancer patients undergoing high-dose chemotherapy. Circulation. 2004; 109:2749-2754.

[PubMed: 15148277] 
12. Nistico C, Bria E, Cuppone F, Fornier M, Sperduti I, Carpino A, Pace A, Cognetti F, Terzoli E. Weekly epirubicin and paclitaxel with granulocyte colony-stimulating factor support in previously untreated metastatic breast cancer patients: a phase II study. Anticancer Drugs. 2007; 18:687-692. [PubMed: 17762397]

13. Dodos F, Halbsguth T, Erdmann E, Hoppe UC. Usefulness of myocardial performance index and biochemical markers for early detection of anthracycline-induced cardiotoxicity in adults. Clin Res Cardiol. 2008; 97:318-326. [PubMed: 18193371]

14. Marchandise B, Schroeder E, Bosly A, Doyen C, Weynants P, Kremer R, Pouleur H. Early detection of doxorubicin cardiotoxicity: interest of Doppler echocardiographic analysis of left ventricular filling dynamics. Am Heart J. 1989; 118:92-98. [PubMed: 2741800]

15. Nagy AC, Tolnay E, Nagykalnai T, Forster T. Cardiotoxicity of anthracycline in young breast cancer female patients: the possibility of detection of early cardiotoxicity by TDI. Neoplasma. 2006; 53:511-517. [PubMed: 17167721]

16. Pudil R, Horacek JM, Strasova A, Jebavy L, Vojacek J. Monitoring of the very early changes of left ventricular diastolic function in patients with acute leukemia treated with anthracyclines. Exp Oncol. 2008; 30:160-162. [PubMed: 18566583] 


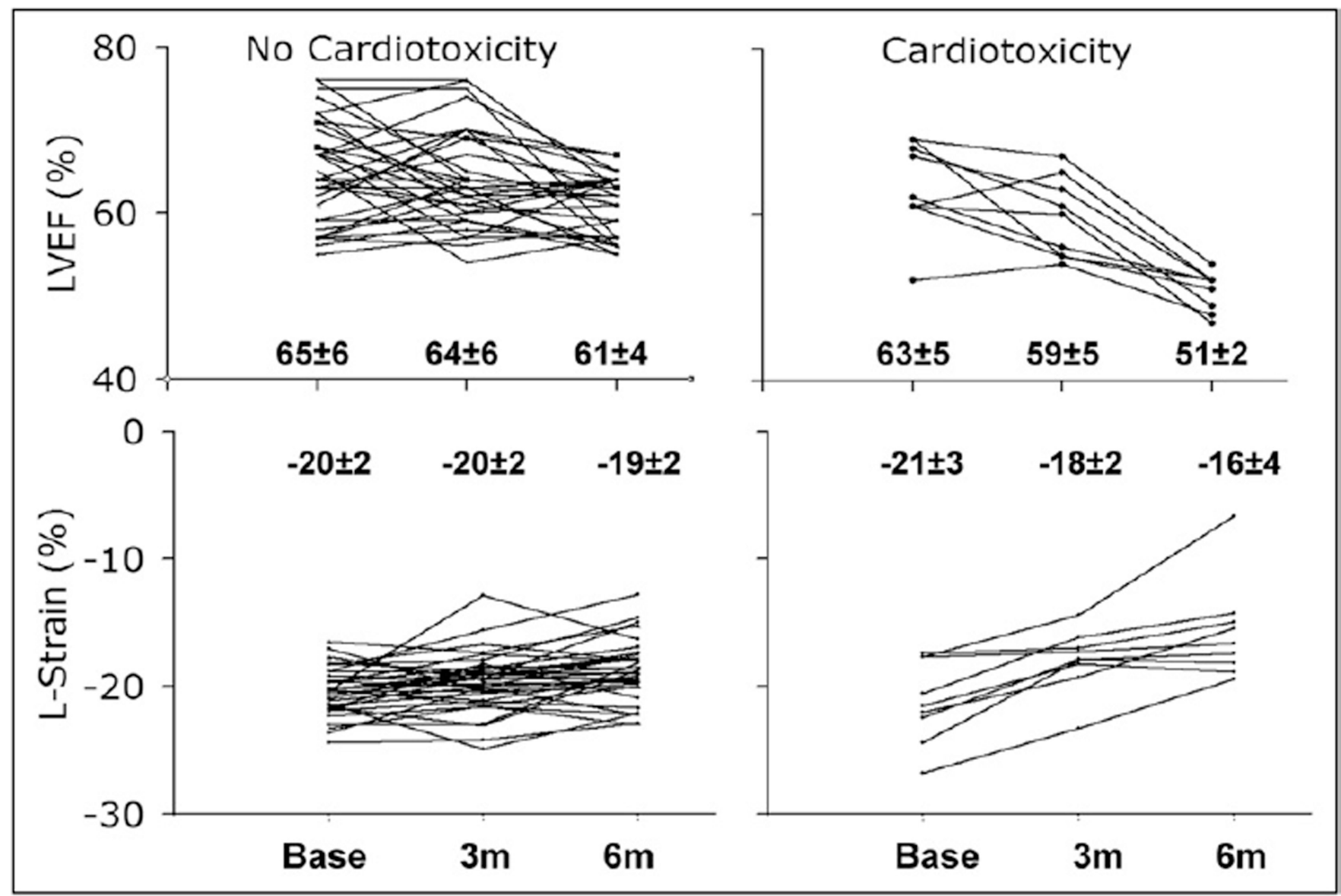

Figure 1.

Individual changes in the $\operatorname{LVEF}(A)$ and longitudinal strain $(B)$ in patients who did not develop (left) and those who developed (right) cardiotoxicity. 
Table 1

Baseline clinical characteristics of patients treated with anthracyclines and trastuzumab who developed or did not develop cardiotoxicity

\begin{tabular}{|c|c|c|c|}
\hline \multirow[b]{2}{*}{ Variable } & \multicolumn{2}{|c|}{ Cardiotoxicity } & \multirow[b]{2}{*}{$\begin{array}{c}\mathbf{p} \\
\text { Value }\end{array}$} \\
\hline & $\begin{array}{c}\text { Yes } \\
(\mathbf{n}=9)\end{array}$ & $\begin{array}{c}\text { No } \\
(\mathbf{n}=\mathbf{3 4})\end{array}$ & \\
\hline Age (years) & $47 \pm 11$ & $49 \pm 10$ & 0.94 \\
\hline Previous treatment with anthracyclines & $2(22 \%)$ & $8(23 \%)$ & 1.00 \\
\hline Dose of anthracyclines & & & 1.00 \\
\hline Doxorubicin $240 \mathrm{mg} / \mathrm{m}^{2}$ & $9(100 \%)$ & $30(88 \%)$ & \\
\hline Epirubicin $300 \mathrm{mg} / \mathrm{m}^{2}$ & $0(0 \%)$ & $4(12 \%)$ & \\
\hline Treatment with taxanes & $9(100 \%)$ & $34(100 \%)$ & 1.00 \\
\hline Radiotherapy & $2(22 \%)$ & $3(9 \%)$ & 0.28 \\
\hline Side of breast cancer & & & 1.00 \\
\hline Right & $3(33 \%)$ & $14(41 \%)$ & \\
\hline Left & $6(67 \%)$ & $19(56 \%)$ & \\
\hline Both & $0(0 \%)$ & $1(3 \%)$ & \\
\hline \multicolumn{4}{|l|}{ Cardiac risk factors } \\
\hline Blood pressure $\geq 140 / \geq 90 \mathrm{~mm} \mathrm{Hg}$ & $2(22 \%)$ & $10(29 \%)$ & 1.00 \\
\hline Total cholesterol >200 mg/dl & $0(0 \%)$ & $8(23 \%)$ & 0.66 \\
\hline Diabetes mellitus & $0(0 \%)$ & $1(3 \%)$ & 1.00 \\
\hline Smoker & $1(11 \%)$ & $5(15 \%)$ & 0.31 \\
\hline \multicolumn{4}{|l|}{ Cardiovascular therapy } \\
\hline Angiotensin-converting enzyme inhibitors & $1(11 \%)$ & $3(9 \%)$ & 0.19 \\
\hline$\beta$ blockers & $0(0 \%)$ & $3(9 \%)$ & 1.00 \\
\hline Body mass index $\left(\mathrm{kg} / \mathrm{m}^{2}\right)$ & $23 \pm 12$ & $26 \pm 4$ & 0.74 \\
\hline Systolic blood pressure $(\mathrm{mm} \mathrm{Hg})$ & $120 \pm 11$ & $122 \pm 18$ & 0.98 \\
\hline Diastolic blood pressure (mm Hg) & $75 \pm 10$ & $74 \pm 9$ & 0.19 \\
\hline Heart rate (beats/min) & $71 \pm 11$ & $69 \pm 9$ & 0.38 \\
\hline
\end{tabular}

Data are expressed as mean \pm SD or as number (percentage). 
Table 2

Clinical, echocardiographic, and biomarker characteristics of 43 patients before and after treatment with anthracyclines and trastuzumab

\begin{tabular}{|c|c|c|c|c|}
\hline Variable & Baseline & 3 Months & 6 Months & $\begin{array}{c}\text { p Value } \\
(\text { ANOVA) }\end{array}$ \\
\hline Systolic blood pressure (mm Hg) & $122 \pm 17$ & $116 \pm 18$ & $119 \pm 15$ & 0.07 \\
\hline Diastolic blood pressure (mm Hg) & $74 \pm 9$ & $72 \pm 10$ & $72 \pm 9$ & 0.33 \\
\hline Heart rate (beats/min) & $69 \pm 10$ & $72 \pm 10$ & $73 \pm 11$ & 0.10 \\
\hline LV end-diastolic volume (ml) & $74 \pm 13$ & $74 \pm 18$ & $76 \pm 8$ & 0.58 \\
\hline LV end-systolic volume (ml) & $26 \pm 7$ & $28 \pm 9$ & $33 \pm 11$ & $<0.0001$ \\
\hline LVEF & $0.65 \pm 0.06$ & $0.63 \pm 0.06$ & $0.59 \pm 0.05$ & $<0.0001$ \\
\hline LV end-diastolic diameter (mm) & $43 \pm 4$ & $43 \pm 3$ & $44 \pm 4$ & 0.16 \\
\hline Relative wall thickness & $0.39 \pm 0.07$ & $0.37 \pm 0.06$ & $0.37 \pm 0.06$ & 0.12 \\
\hline Longitudinal strain (\%) & $20.5 \pm 2.2$ & $19.3 \pm 2.4^{*}$ & $18.4 \pm 3$ & $<0.0001$ \\
\hline Radial strain (\%) & $55 \pm 12$ & $52 \pm 12$ & $46 \pm 14$ & 0.02 \\
\hline Circumferential strain (\%) & $18 \pm 4$ & $15 \pm 4 \%$ & $14 \pm 3$ & 0.001 \\
\hline Left atrial volume (ml) & $32 \pm 12$ & $32 \pm 9.5$ & $32 \pm 11.5$ & 0.91 \\
\hline Mitral E-wave filling velocity $(\mathrm{cm} / \mathrm{s})$ & $77 \pm 17$ & $75 \pm 19$ & $76 \pm 18$ & 0.36 \\
\hline Mitral A-wave filling velocity $(\mathrm{cm} / \mathrm{s})$ & $67 \pm 15$ & $65 \pm 15$ & $64 \pm 19$ & 0.69 \\
\hline Mitral E-wave filling velocity/mitral A-wave filling velocity & $1.2 \pm 0.3$ & $1.2 \pm 0.3$ & $1.3 \pm 0.4$ & 0.44 \\
\hline Peak early diastolic mitral annular velocity $(\mathrm{cm} / \mathrm{s})$ & $12.5 \pm 2.8$ & $11.8 \pm 3.1^{\dagger}$ & $11 \pm 2.5$ & 0.004 \\
\hline Peak late diastolic mitral annular velocity $(\mathrm{cm} / \mathrm{s})$ & $10 \pm 3$ & $10 \pm 3$ & $9 \pm 2$ & 0.11 \\
\hline Mitral E-wave filling velocity/peak early diastolic mitral annular velocity & $6.7 \pm 2$ & $6.8 \pm 2.6$ & $7 \pm 2.3$ & 0.59 \\
\hline hsTnI $(\mu \mathrm{g} / \mathrm{L})$ & $0.00 \pm 0.01$ & $0.04 \pm 0.13$ & $0.02 \pm 0.07$ & 0.09 \\
\hline NT-proBNP (pg/ml) & $103 \pm 73$ & $98 \pm 105$ & $91 \pm 114$ & 0.12 \\
\hline
\end{tabular}

Data are expressed as mean $\pm \mathrm{SD}$.

p $<0.001$.

$t_{\mathrm{p}}<0.02$.

${ }_{\mathrm{p}}^{*}=0.001$ versus baseline.

ANOVA $=$ analysis of variance; $L V=$ left ventricular. 


\section{Table 4}

Sensitivity, specificity, and positive and negative value of the predictors of cardiotoxicity

\begin{tabular}{lcccc}
\hline Predictor & Sensitivity & Specificity & $\begin{array}{c}\text { Positive Predictive } \\
\text { Value }\end{array}$ & $\begin{array}{c}\text { Negative Predictive } \\
\text { Value }\end{array}$ \\
\hline $10 \%$ decrease in longitudinal strain & $7 / 9(78 \%)$ & $27 / 34(79 \%)$ & $7 / 14(50 \%)$ & $27 / 29(93 \%)$ \\
Elevated hsTnI at 3 months & $6 / 9(67 \%)$ & $28 / 34(82 \%)$ & $6 / 12(50 \%)$ & $28 / 31(90 \%)$ \\
$10 \%$ decrease in longitudinal strain and elevated hsTnI at 3 & $5 / 9(55 \%)$ & $33 / 34(97 \%)$ & $5 / 6(83 \%)$ & $33 / 37(89 \%)$ \\
months & & & & \\
$10 \%$ decrease in longitudinal strain or elevated hsTnI at 3 months & $8 / 9(89 \%)$ & $22 / 34(65 \%)$ & $8 / 20(40 \%)$ & $22 / 23(97 \%)$ \\
\hline
\end{tabular}

